\title{
Coupling Between Finite Ground Coplanar Waveguides Embedded in Polyimide Layers for 3D-MMICs on Si
}

\author{
George E. Ponchak ${ }^{1}$, John Papapolymerou ${ }^{2}$ and Emmanouil M. Tentzeris ${ }^{3}$
}

1. NASA Glenn Research Center, Cleveland, $\mathrm{OH} 44135$; george.ponchak@grc.nasa.gov

2. Dept. of Electrical and Computer Engineering, The University of Arizona, Tucson, AZ 85721

3. School of Electrical and Computer Engineering, Georgia Inst. of Technology, Atlanta, GA 30332

\begin{abstract}
Three-dimensional circuits built upon multiple layers of polyimide are required for constructing $\mathrm{Si} / \mathrm{SiGe}$ monolithic microwave/mm-wave integrated circuits on low resistivity $\mathrm{Si}$ wafers. However, the closely spaced transmission lines are susceptible to high levels of cross-coupling, which degrades the overall circuit performance. In this paper, theoretical and experimental results on coupling of Finite Ground Coplanar (FGC) waveguides embedded in polyimide layers are presented for the first time. These results show that FGC lines have approximately $8 \mathrm{~dB}$ lower coupling than coupled Coplanar Waveguides. Furthermore, it is shown that the forward and backward coupling characteristics for FGC lines do not resemble the coupling characteristics of other transmission lines such as microstrip.
\end{abstract}

Index terms - Coplanar Waveguide, Finite Ground Coplanar Waveguide, Coupling, Crosstalk

\section{INTRODUCTION}

There is a rapidly expanding market for $\mathrm{Si}$ Microwave/mm-wave Monolithic Integrated Circuits (MMICs) fabricated in standard CMOS foundries to replace GaAs MMICs and ceramic circuits (e.g. Alumina) in wireless communication systems, phased array radars, and other applications where the circuit cost is a major factor in determining the system cost. However, microwave passive elements and transmission lines placed directly on standard CMOS grade Si substrate have low quality factors (high attenuation), which necessitates novel transmission line structures [1] that are typically embedded in polyimide that is deposited over the Si substrate. An alternative is to use high resistivity Si substrates, but that increases the cost relative to the CMOS grade wafers. Moreover, highly integrated systems that include the RF circuits, MEMS circuits and sensors, digital data processing circuits, and bias control circuits on a single chip or within a single package also rely on multiple layers of polyimide to construct three dimensional circuits that are smaller than would normally be possible.

One of the first transmission lines developed for low resistivity $\mathrm{Si}$ wafers was thin film microstrip line (TFMS); TFMS is comprised of a metal ground plane deposited on top of the $\mathrm{Si}$ wafer, a polyimide or similar material that is deposited over the ground plane and serves as the microstrip substrate, and finally the definition of the microstrip line. Because the ground plane shields all of the electromagnetic fields from the $\mathrm{Si}$ and the polyimide layer is thin, typically less than $20 \mu \mathrm{m}$, TFMS solved the problem of high attenuation and smaller sized circuits [2]. Coupling between two parallel TFMS lines embedded in polyimide layers was recently studied and presented [3]. Results showed that acceptable levels of coupling can be obtained if shielding structures are used.

However, Coplanar Waveguides (CPW) and Finite Ground Coplanar (FGC) lines are often preferred in microwave circuit design over microstrip lines due to the easy integration of both series and shunt elements without via holes and costly back side processing, the need for only a single metallization layer, and easier component integration using flip-chip attachment. FGC lines are an excellent solution for multi-layer microwave circuits because they do not excite parasitic modes and support an almost pure TEM mode of propagation up to $120 \mathrm{GHz}$, making circuit design extremely easy [4]. Coupling between adjacent FGC lines on high resistivity Si substrate has been characterized [5], and results show that the FGC lines have lower coupling than CPW lines.

In this paper, an initial evaluation of the coupling between FGC lines embedded in polyimide layers built upon both high-resistivity and CMOS grade $\mathrm{Si}$ substrates is presented for the first time. Experimental measurements and computer simulations based on the Method of Moments are used for this evaluation. Coupling between FGC lines embedded in polyimide is compared to coupling between FGC lines on high resistivity silicon and TFMS lines embedded in polyimide.

\section{Circuit Description}

Figure 1 shows a cross-sectional view of two adjacent FGC lines on a polyimide layer on top of a Si substrate. In this case the two lines are on the same polyimide layer and the distance between them is D. The center conductor width is $\mathrm{S}$, the slot line width is $\mathrm{W}$, and the ground plane width is B. The initial investigation on coupling effects will focus on adjacent lines of the same geometry and, thus, of the same characteristic impedance. Coupling between lines of different characteristic impedance will also be analyzed though at a later time. For this paper, coupling between 
FGC lines with $\mathrm{S}=42 \mu \mathrm{m}$ and $\mathrm{W}=6 \mu \mathrm{m}, \mathrm{S}=42 \mu \mathrm{m}$ and $\mathrm{W}=24 \mu \mathrm{m}$, and $\mathrm{S}=20 \mu \mathrm{m}$ and $\mathrm{W}=10 \mu \mathrm{m}$ that have characteristic impedances, Zc, of 54,75 , and $81 \Omega$ respectively are characterized. For each FGC line, coupling is characterized for $\mathrm{D}=0$ to $30 \mu \mathrm{m}$ and $\mathrm{B}=\mathrm{S}, 2 \mathrm{~S}$, and $3 \mathrm{~S}$. The thickness of the polyimide layer is equal to $20 \mu \mathrm{m}$, while the thickness of the Si substrate (high-resistivity or CMOS grade) is approximately $500 \mu \mathrm{m}$. Not shown for clarity is a second polyimide layer of $3 \mu \mathrm{m}$ thickness that covers the structure shown in Figure 1; this thin polyimide layer supports bridges that connect the ground planes of each FGC line to suppress the parasitic slotline mode.

The circuit for characterizing coupling between the FGC lines is shown in Figure 2. The four-port circuit has probe pads oriented so that each port may be probed simultaneously. The coupling region, or the section of parallel transmission lines labeled L in Figure 2, is 7500 $\mu \mathrm{m}$ long.

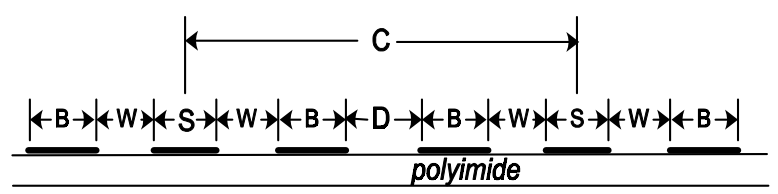

Si

Figure 1: Cross-sectional cut of adjacent FGC lines embedded in polyimide.

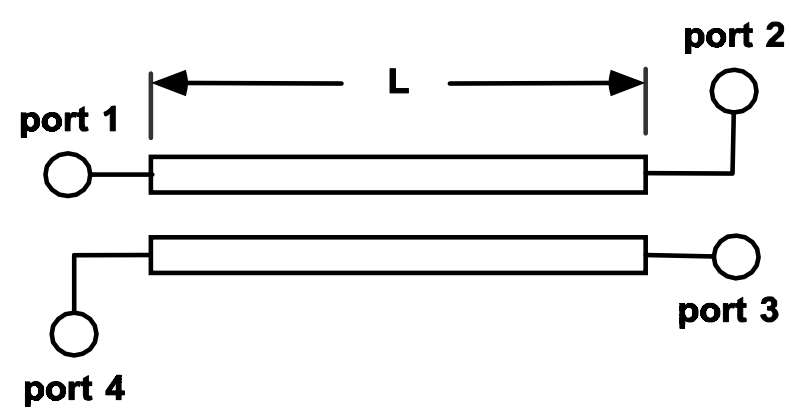

Figure 2: Schematic of coupled line structures used to characterize coupling.

\section{CIRCUIT FABRICATION AND CHARACTERIZATION}

For the theoretical characterization of the FGC coupling a commercially available software tool based on the $2.5 \mathrm{D}$ Method of Moments technique (Sonnet) is used. In the simulations, each FGC line is assumed to have perfect electric conductors, while the $\mathrm{Si}$ substrate is modeled with its dielectric constant, dielectric loss and conductivity based on its resistivity value $(\sigma=100 \mathrm{~S} / \mathrm{m}$ for the CMOS wafer and $\sigma=0$ for the high-resistivity wafer). For the polyimide layer, no dielectric loss is taken into account. The excitation of the four different ports is based on the coplanar mode only.

The four-port circuits are fabricated on a 1 and $2500 \Omega$ $\mathrm{cm} \mathrm{Si}$ wafers. Twenty microns of Dupont PI-1111 polyimide, which has a relative permittivity of 2.8 , is spun onto the Si wafer in a single layer and cured. After curing, $200 \AA$ of $\mathrm{Ti}$ and $1.5 \mu \mathrm{m}$ of $\mathrm{Au}$ are electron beam evaporated onto the wafer and defined through standard lift-off processing techniques. To build the bridges required to suppress the slotline mode, $3 \mu \mathrm{m}$ of PI-2611 polyimide, relative permittivity of 3.12 , is spun onto the wafer in a single step and cured. $\mathrm{Ni}$ is then evaporated onto the polyimide to serve as a mask for the $\mathrm{O}_{2} / \mathrm{CF}_{4}$ reactive ion etching (RIE) of via holes through the second polyimide layer. After via holes are etched and the Ni removed, the bridges are defined by lift-off processing of $200 \AA$ of Ti and $1.5 \mu \mathrm{m}$ of Au. The ground bridges are spaced approximately $1500 \mu \mathrm{m}$ along each FGC line.

Measurements are made on a vector network analyzer from 2 to $50 \mathrm{GHz}$. A Thru/Reflect/Line (TRL) calibration is implemented with MULTICAL [6], a TRL software program, using four delay lines of $850,1700,3500$, and $10000 \mu \mathrm{m}$ and a short circuit reflect fabricated on the same substrate as the circuits. Two of the four ports are terminated in $50 \Omega$ loads built into especially designed RF probes. Finally, to eliminate parasitic microstrip modes, a quartz plate is placed between the $\mathrm{Si}$ substrate and the wafer chuck during testing.

\section{RESULTS}

The measured results for a two coupled line structures are shown in Figures $3 \mathrm{a}$ and $3 \mathrm{~b} . \mathrm{S}_{21}$ is not plotted because the coupling is very low in all cases measured and $\mathrm{S}_{21}$ simply shows the attenuation of the $7500 \mu \mathrm{m}$ long FGC line. First, the backward coupling defined as $-20 \log \left|\mathrm{S}_{41}\right|$ and the forward coupling defined as $-20 \log \left|S_{31}\right|$ for the FGC lines with $\mathrm{D}=0$ have the typical characteristics of monotonically increasing forward coupling with frequency and backward coupling that is periodic with frequency as shown in Figure 3a. However, when a small gap is introduced between the ground planes, the characteristics are not similar to results presented in the literature for coupled transmission lines such as microstrip. In Figure 3b, which shows the coupling for $\mathrm{D}=6 \mu \mathrm{m}$, it is seen that for low frequency, less than $10 \mathrm{GHz}$, FGC coupling almost behaves as expected, but this is a special case of coupled lines that are electrically short. At higher frequency, the measured forward and backward coupling has the same characteristics, which appear to show coupling increasing with frequency, although not monotonically. These results are typical of the other measured circuits and are in qualitative agreement with the Sonnet simulations. Sonnet simulations also show that the characteristics shown in Figure $3 \mathrm{~b}$ are not an artifact of substrate modes. Rather, it is believed that the forward and backward coupling when $\mathrm{D}>0$ is due to indirect coupling as described by Schelkunoff [7]. This indirect coupling is a result of a parasitic mode due to the ground planes of each FGC line that create a third transmission line.

More importantly, the forward and backward coupling is reduced by approximately $8 \mathrm{~dB}$ across the entire frequency band when $\mathrm{D}>0$. Figures 4-6 summarizes this reduction in coupling by plotting the maximum measured coupling for $\mathrm{f}<10 \mathrm{GHz}$ for each of the structures characterized. It is seen that there is a large reduction in 
coupling when $\mathrm{D}>0$, even when $\mathrm{D}$ is as small as $6 \mu \mathrm{m}$, which implies that the nature of the coupling changes when $\mathrm{D}>0$. Also, it is seen that for the electrically short, coupled line sections shown in Figures 4-6, the backward coupling is approximately $5 \mathrm{~dB}$ higher than the forward coupling. However, Figure $3 \mathrm{~b}$ shows that this is not true for higher frequencies where the forward and backward coupling have similar values.

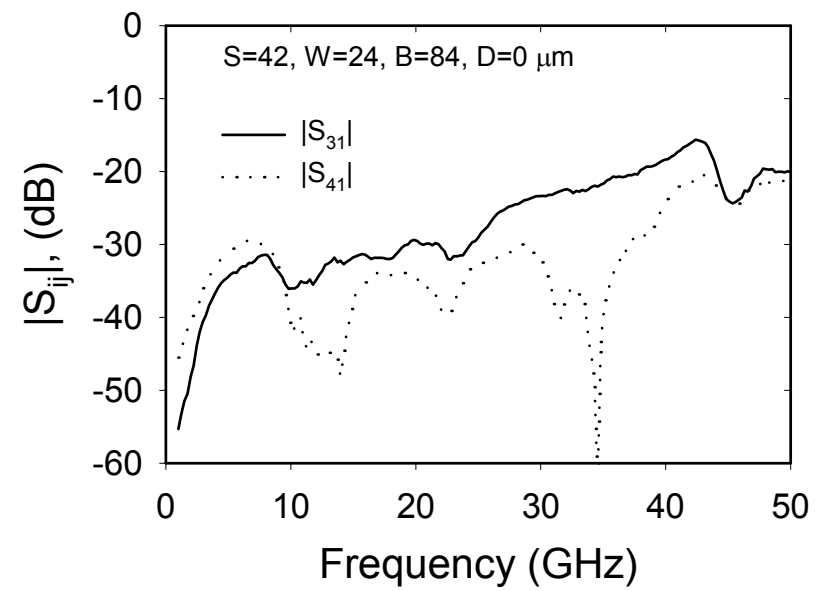

(a)

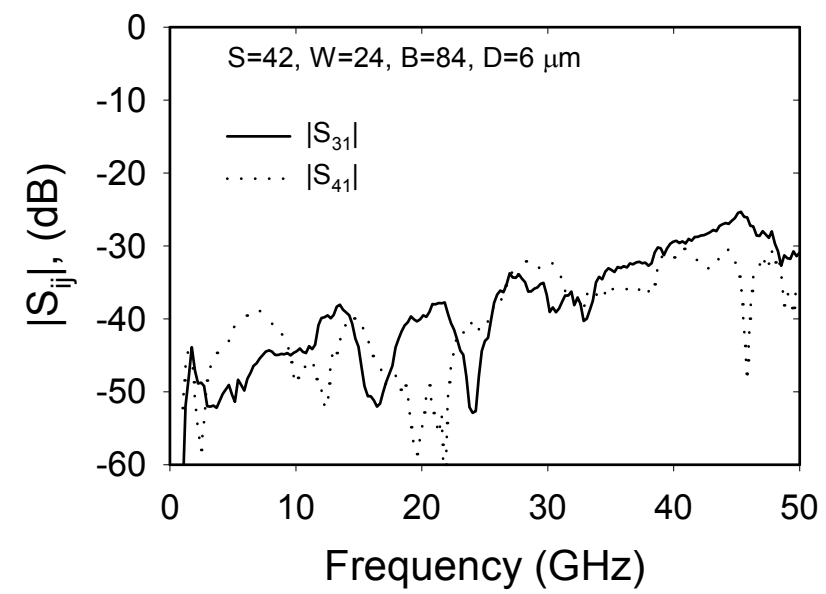

(b)

Figure 3: Measured coupling parameters for FGC lines fabricated on high resistivity $\mathrm{Si}$ with $\mathrm{S}=42, \mathrm{~W}=\mathbf{2 4}, \mathrm{B}=84$, and (a) $\mathrm{D}=0$, and (b) $\mathrm{D}=6$ $\mu \mathrm{m}$.

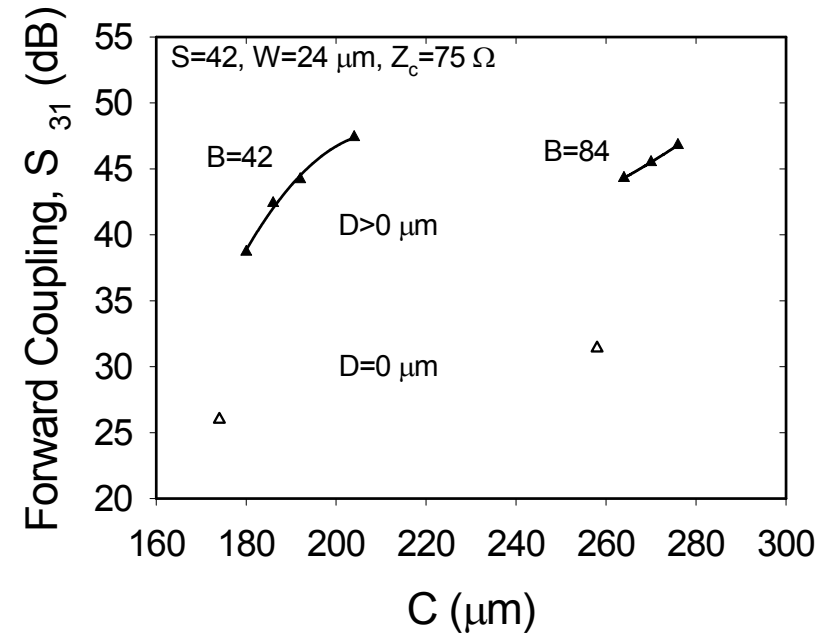

(a)

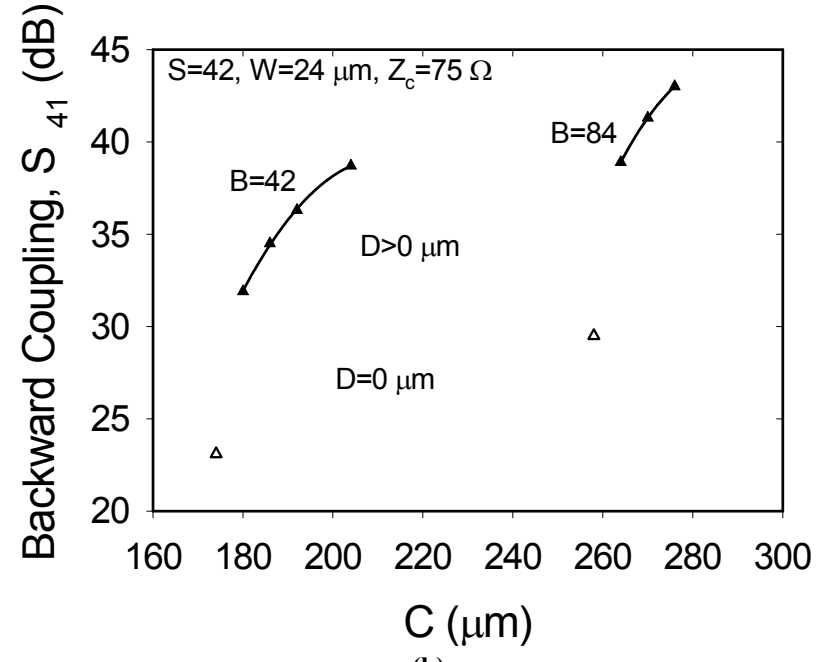

(b)

Figure 4: Summarized (a) forward coupling and (b) backward coupling for FGC lines with $S=42$ and $W=24 \mu \mathrm{m}$ fabricated on high resistivity $\mathrm{Si}$. Coupling shown is the maximum coupling measured for f $<10$ GHz.

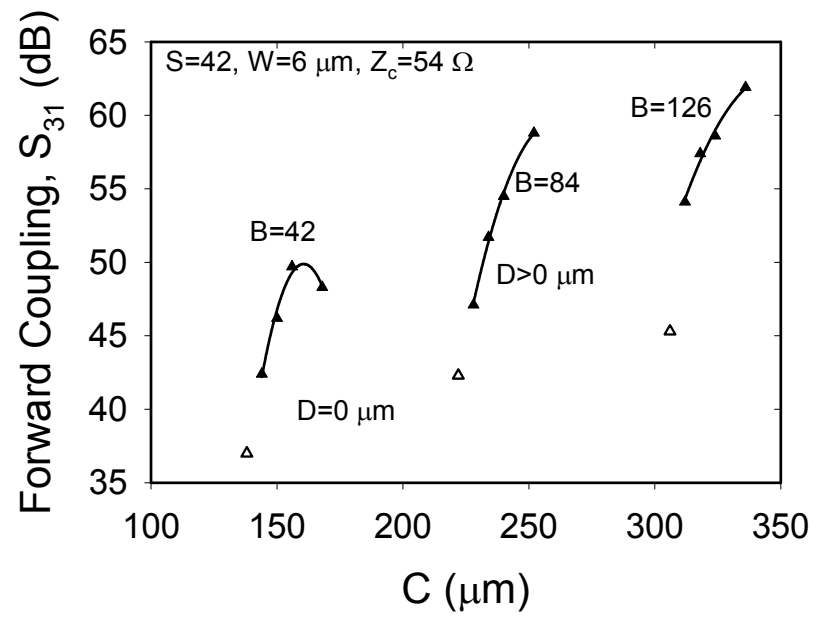

(a)

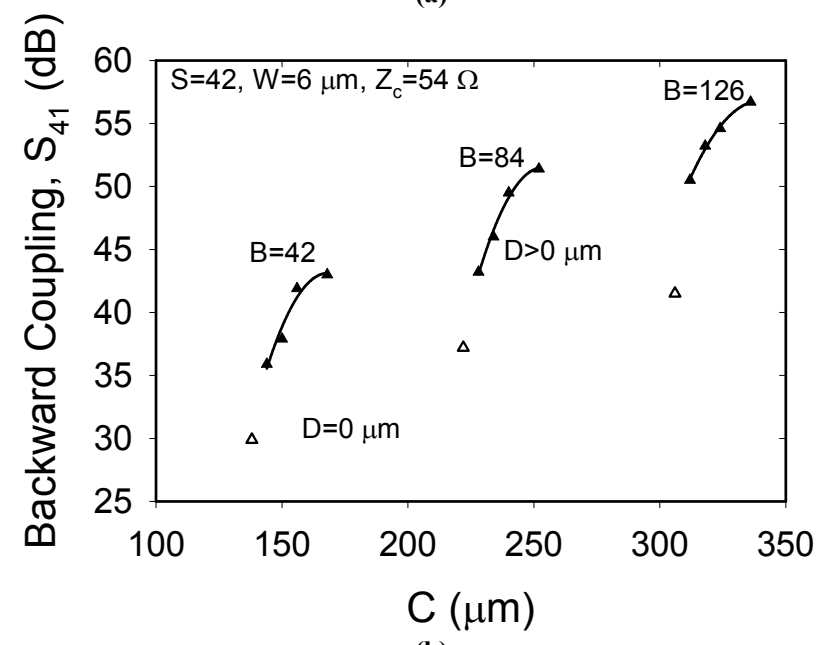

(b)

Figure 5: Summarized (a) forward coupling and (b) backward coupling for FGC lines with $S=42$ and $W=6 \mu \mathrm{m}$ fabricated on high resistivity $\mathrm{Si}$. Coupling shown is the maximum coupling measured for f $<10$ GHz. 


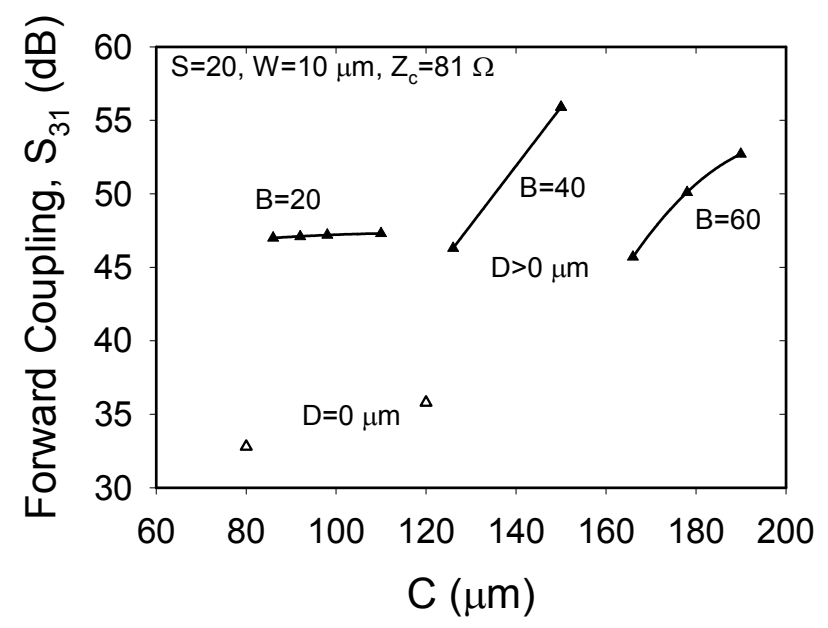

(a)

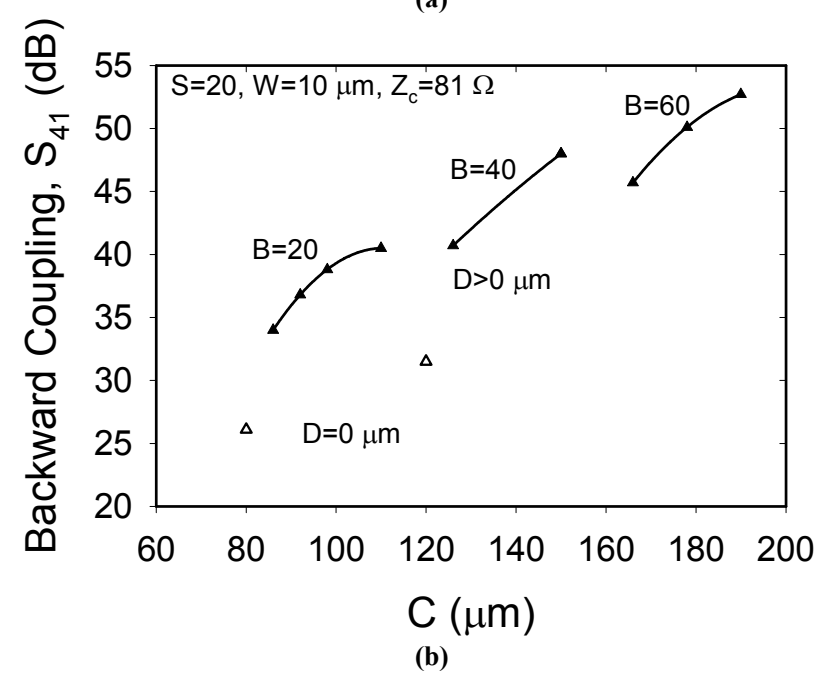

Figure 6: Summarized (a) forward coupling and (b) backward coupling for $F G C$ lines with $S=20$ and $W=10 \mu \mathrm{m}$ fabricated on high resistivity $\mathrm{Si}$. Coupling shown is the maximum coupling measured for f $<10$ GHz.

By comparing the coupling of FGC lines embedded in polyimide presented in this paper to coupling between FGC lines directly on high resistivity silicon presented in [5], it is seen that the coupling is lower for the embedded FGC lines. Using results presented in [3], it is also seen that coupling between FGC lines embedded in polyimide is lower than coupling between thin film microstrip lines embedded in polyimide, and even if shielding structures are used with the thin film microstrip, the coupling is comparable between the two lines.

\section{CONCLUSIONS}

This paper shows for the first time the coupling between finite ground coplanar waveguides embedded in polyimide for 3D-MMICs. The results show embedded FGC lines have low coupling, and that there is a large reduction in coupling when there is a gap between the ground planes of each coplanar waveguide. Measured results confirmed by theoretical simulations also show that the nature of the coupling is different for the FGC lines compared to thin film microstrip lines.

\section{REFERENCES}

[1] G. E. Ponchak, "RF transmission lines on silicon substrates," $29^{\text {th }}$ European Microwave Conference Digest, pp. 158-161, Munich, Germany, October 1999.

[2] G. E. Ponchak and A. N. Downey, "Characterization of thin film microstrip lines on polyimide," IEEE Trans. Components, Packaging, and Manufacturing Tech. -Part B, Vol. 21, No. 2, pp. 171-176, May 1998.

[3] G. E. Ponchak, E. Tentzeris, and J. Papapolymerou, "Coupling between microstrip lines embedded in polyimide layers for 3DMMICs on Si," to be published in the 2001 IEEE Int. Microwave Symposium Digest, Phoenix, AZ, May 2001.

[4] F. Brauchler, S. Robertson, J. East, and L. Katehi, "W-Band finite ground coplanar line circuit elements," 1999 IEEE Int. Microwave Symposium Digest, pp. 1845-1848, San Francisco, CA, June 1996.

[5] G. E. Ponchak, L. P. B. Katehi, and E. Tentzeris, "Characterization of the coupling between adjacent FGC waveguides," The Int. Journal of Microcircuits and Electronic Packaging, Vol. 20, No. 4, pp. 587-592, Fourth Quarter 1997.

[6] R. B. Marks, "A multilane method of network analyzer calibration," IEEE Trans. Microwave Theory Tech., Vol. 39, pp. 1205-1215, July 1991.

[7] S. A. Schelkunoff and T. M. Odarenko, "Crosstalk between coaxial transmission lines," Bell System Technical Journal, Vol. 16, pp. 144-164, 1937. 